\title{
Deep science strikes gold after latest site is named
}

In the world of underground science, space is tight, and getting tighter. So scientists across the globe are welcoming a proposal for a new US facility that could help relieve the growing subterranean real-estate crisis.

On 10 July, the US National Science Foundation (NSF) announced that it had selected the abandoned Homestake gold mine near Lead, South Dakota, as the preferred site for a US\$500-million Deep Underground Science and Engineering Laboratory. If fully funded, the mine will be developed into a sprawling underground campus - the deepest yet - where geologists, microbiologists and physicists can ply their trade.

It is physicists in particular who want the space, and who have been driving the push for the new lab. For decades, they have travelled to road tunnels and abandoned mines to build experiments that must be shielded from cosmic radiation. Only a handful of locations can host the searches, and many are becoming overcrowded, says Eugenio Coccia, director of the world's largest underground facility, the 180,000-cubic-metre Gran Sasso National Laboratory near L'Aquila, Italy. "There is no more empty space," he says.

In the United States, the situation is even worse, says Bernard Sadoulet, a physicist at the University of California, Berkeley. America's only major underground facility is at the
Soudan mine in Minnesota, and Sadoulet, who co-chaired a review of underground science for the NSF, says his committee received around 80 letters of interest in the new lab.

Then there is the problem of depth: the Soudan mine is 710 metres deep, but most of the newer experiments need to go deeper. Sadoulet's own experiment at Soudan probes dark matter - particles that interact only rarely but make up roughly a quarter of the mass of the Universe (see page 240). He says the next generation will be ready before Homestake and will be sited at the 2,070-metre-deep Sudbury Underground Laboratory in Ontario, Canada. Such physics is being driven ever deeper underground. As particle accelerators increase in size and cost, rare-event physics of the sort that can be done only beneath thousands of metres of rock is seen as an increasingly attractive means of probing big questions. For instance, a phenomenon known as neutrinoless double-beta decay, an extremely rare event that occurs during the decays of some nuclei, could prove that neutrinos are their own antiparticle. Such a finding would have implications for the standard model of particle physics, and could explain why there is more matter than antimatter in the Universe today.

The search for these rare processes typically requires that detectors sit for years gathering just a handful of events. With each generation,

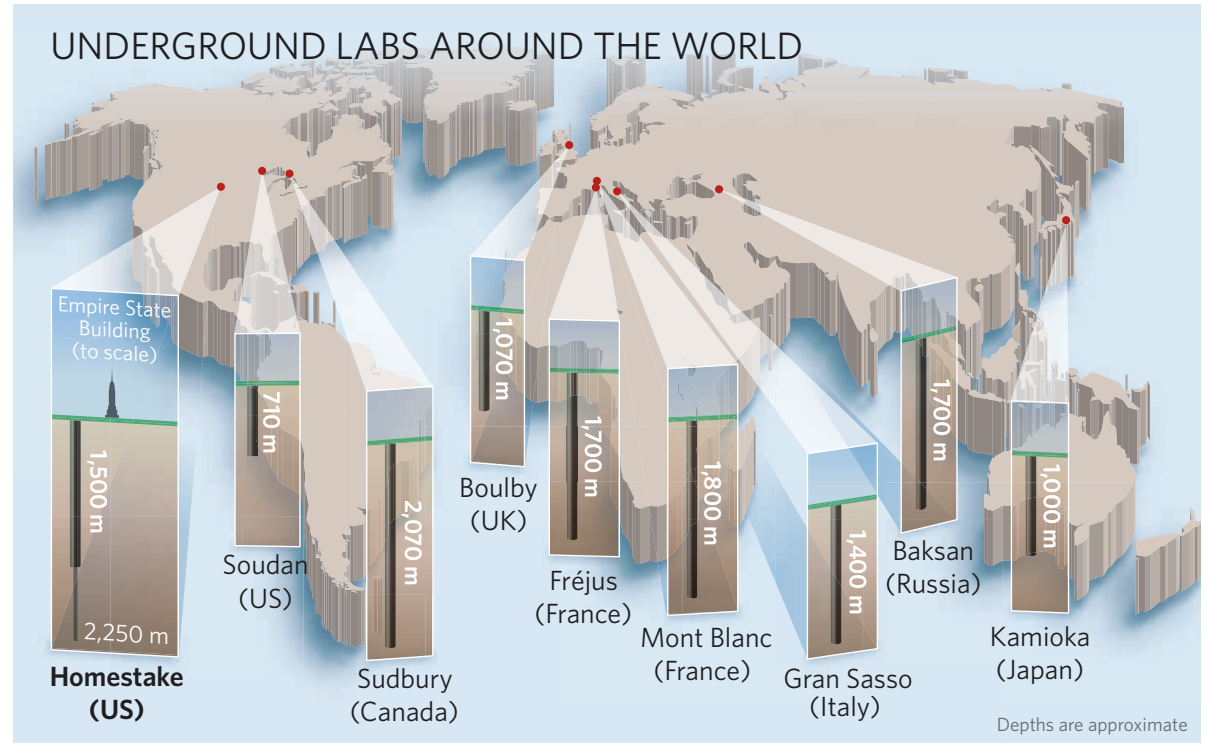

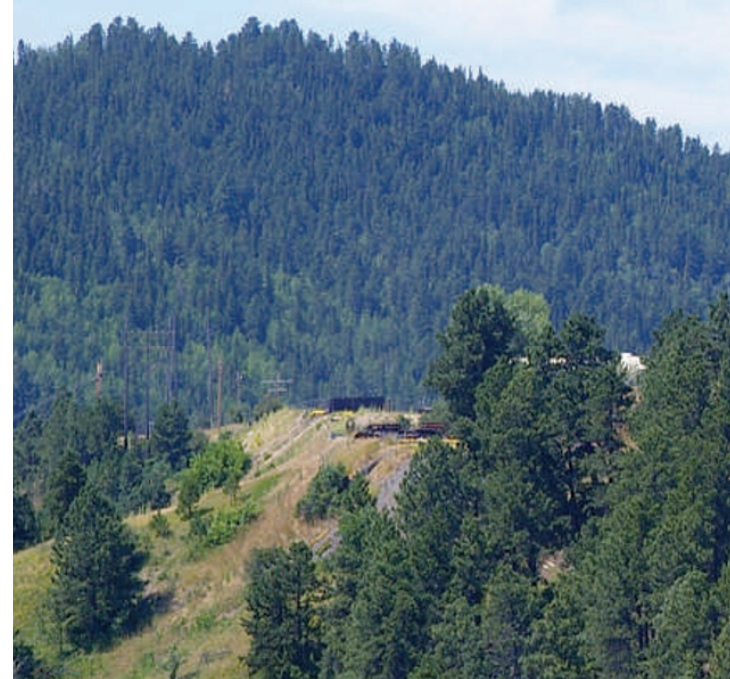

the detectors get bigger and the experiments get longer. Thus, many existing underground laboratories are planning expansions. Proposals are on the table to expand the Fréjus laboratory in France in 2012, says Gilles Gerbier, a physicist at the French Atomic Energy Commission in Saclay. And the Kamioka Observatory in Japan, which hosts the massive Super-Kamiokande neutrino detector, is also digging new spaces for a dark-matter search and double-beta-decay experiment, according to Yoichiro Suzuki, the observatory's director.

The need for increased sensitivity of such experiments also sends them deeper, because each successive metre of rock gives more effective shielding from disruptive cosmic rays. The world's two deepest laboratories, Mont Blanc in France and the Sudbury mine in Canada (see graphic), are cramped. Even after an expansion at Sudbury planned for completion by 2008 , there will be room for only four large experiments, says Arthur McDonald, a physicist at Queen's University in Kingston, Ontario. "We've had more letters of interest than we've space to house the experiments," he says.

Homestake would also provide opportunities for geologists and microbiologists. Geologists could use it to study how rock behaves under pressure; such information may help to understand earthquakes. Meanwhile, microbiologists see the lab as an opportunity to study organisms that live far beneath Earth's surface. "Most of these organisms don't depend on oxygen to survive," says Tullis Onstott, a geomicrobiologist at Princeton University in New Jersey. What nutrients they need, and how they obtain them, could provide clues to how life began, he adds. 
